\title{
Cost-effectiveness of early responders versus early nonresponders to atypical antipsychotic therapy
}

\author{
Xiaomei Peng' \\ Haya Ascher-Svanum' \\ Douglas E Faries' \\ Virginia L Stauffer' \\ Sara Kollack-Walker' \\ Bruce J Kinon' \\ John M Kane ${ }^{2}$
}

'Eli Lilly and Company, Indianapolis, Indiana, USA; ${ }^{2}$ Zucker Hillside

Hospital, Department of Psychiatry, Glen Oaks, New York, USA
Correspondence: Xiaomei Peng

Lilly Corporate Center,

Indianapolis, IN 46285, USA

$\mathrm{Tel}+\mathrm{I} 3 \mid 74339534$

Fax + I 3172776930

Email x_peng@lilly.com
This article was published in the following Dove Press journal:

ClinicoEconomics and Outcomes Research

19 April 201I

Number of times this article has been viewed

Background: To compare the cost-effectiveness of treating early responders versus early nonresponders to an atypical antipsychotic (risperidone) and the cost-effectiveness of treating early nonresponders maintained on risperidone versus those switched to olanzapine.

Methods: This post hoc analysis used data from a randomized, double-blind, 12-week schizophrenia study (Study Code: HGMN, n =628). Participants were initially assigned to risperidone therapy. Early response was defined as a $\geq 20 \%$ improvement on the Positive and Negative Syndrome Scale (PANSS) total score from baseline to two weeks. Early responders continued on risperidone, whereas early nonresponders were randomized in a double-blind manner to continue on risperidone or switch to olanzapine for 10 additional weeks. Early responders and early nonresponders maintained on risperidone were compared for health-state utilities (benefits) and total cost over the 12-week study; early nonresponders maintained on risperidone or switched to olanzapine were compared from randomization (10-week period). Utilities were derived from the PANSS and adverse events. Mixed models were used to assess group differences in utilities. Treatment costs were calculated based on health states. Incremental cost-effectiveness ratios were then utilized to compare treatment groups.

Results: Early responders to risperidone had significantly greater total utility and lower total treatment costs than early nonresponders to risperidone. Compared with early nonresponders who continued on risperidone, those who were switched to olanzapine had significantly higher total utility scores at endpoint and numerically lower total treatment costs, reflecting significantly lower nonmedication treatment costs, even though medication costs were significantly higher compared with generic risperidone.

Conclusion: Treatment of early responders was more cost-effective than treatment of early nonresponders to atypical antipsychotic therapy. Switching early nonresponders to olanzapine resulted in improved treatment effectiveness, met the criteria for some dominance, and appeared modestly more cost-effective than maintaining treatment with generic risperidone.

Keywords: cost-benefit analysis, olanzapine, risperidone, treatment outcome, schizophrenia

\section{Introduction}

Before switching a patient with schizophrenia to a new antipsychotic medication, expert consensus guidelines recommend that clinicians wait 4-8 weeks before changing a patient's antipsychotic regimen, ${ }^{1,2}$ in part due to the possibility of a delayed onset of action. However, a comprehensive meta-analysis and several post hoc analyses have tested and rejected the notion of a delayed response to antipsychotics. ${ }^{3-7}$

A number of studies have demonstrated that early nonresponse to antipsychotics is a strong predictor of subsequent nonresponse to continued treatment with the same antipsychotic. In one study, Correll et al found that every patient whose symptoms 
had not improved by $20 \%$ after one week of treatment were not responders after four weeks. ${ }^{3}$ In a larger analysis, Kinon et al pooled five randomized, double-blind trials of antipsychotics in the treatment of schizophrenia, and found that a lack of response at two weeks was a robust predictor of poor response at 12 weeks. ${ }^{4}$ These findings were extended in a naturalistic trial, where early nonresponders were found not only to have worse clinical outcomes, but worse functional and economic outcomes as well. ${ }^{5}$

All of these studies were retrospective in nature, but a recent 12 -week clinical trial $(\mathrm{HGMN})^{8,9}$ was specifically designed to examine the early response hypothesis in an a priori manner. This study confirmed previous clinical and functional findings demonstrating poor outcomes for individuals with schizophrenia who were not early responders to an atypical antipsychotic (risperidone). HGMN also examined whether early nonresponders who continue on the initial antipsychotic (risperidone) after the first two weeks of therapy differ in treatment outcomes from early nonresponders who were randomized in a double-blind manner to another antipsychotic (olanzapine). Findings indicated that early nonresponders who switched to olanzapine had a small but significantly greater reduction in symptoms at endpoint relative to early nonresponders who remained on risperidone. However, the early responders to risperidone had significantly greater symptom reductions than either group of early nonresponders. The economic implications of these findings have not yet been studied.

The objectives for this analysis were two-fold: to compare the cost-effectiveness of treating early responders versus early nonresponders to an atypical antipsychotic (risperidone) over a 12-week period, and to compare the cost-effectiveness of treating early nonresponders to risperidone who were randomized in a double-blind manner to either continue on risperidone or to switch to olanzapine for an additional 10 weeks of therapy.

\section{Materials and methods}

\section{Data source}

Data for this post hoc analysis came from a 12-week, randomized, double-blind, flexible dose HGMN trial that was explicitly designed to validate whether early responders to risperidone had greater reductions in psychopathology relative to early nonresponders to risperidone, and secondly, to investigate the effects of switching early risperidone nonresponders to olanzapine or continuing them on risperidone. ${ }^{8}$ HGMN included adult patients aged $18-65$ years who met the Diagnostic and Statistical Manual of Mental Disorders, Fourth
Edition (DSM-IV) ${ }^{10}$ criteria for schizophrenia, schizoaffective disorder, or schizophreniform disorder. The multicenter trial started in May 2006, ended in December 2007, and was conducted at 64 centers in three countries. Before undergoing any study procedure, the eligible patients were given a complete description of the study and provided written informed consent. The study was conducted in accordance with the Declaration of Helsinki and was approved by the Ethics Committee from each participating institution. The clinical trial was registered with ClinicalTrials.gov (identifier NCT00337662).

During the 2-5-day initial screening period (study period 1), study participants were assessed to ensure that they met the inclusion and exclusion criteria. Beyond age and diagnosis, the primary inclusion criteria included a score $\geq 45$ on the Brief Psychiatric Rating Scale (BPRS), ${ }^{11}$ total score extracted from the Positive and Negative Syndrome Scale (PANSS) $^{12}$ a score of $\geq 4$ (moderate) on at least two BPRS items: conceptual disorganization, suspiciousness, hallucinatory behavior, and unusual thought content, a minimum Clinical Global Impressions-Severity scale ${ }^{13}$ rating of 4 (moderately ill), and, within two weeks of the first visit, patients needed to have experienced an exacerbation of their illness that required an increased level of psychiatric care. Patients for whom risperidone or olanzapine was contraindicated, and those who had been hospitalized for more than two weeks preceding the first visit or had another serious or unstable medical condition were excluded.

During study period 2, patients began flexible dose therapy with risperidone $2-6 \mathrm{mg} /$ day. Previous antipsychotic medications were discontinued abruptly, and risperidone was initiated with a dosing schedule of $2 \mathrm{mg} /$ day on day $1,4 \mathrm{mg} /$ day on days 2-7, and flexible dosing 2-6 mg/day after day 7 .

At week 2, patients who met the a priori definition for early nonresponse $(<20 \%$ improvement on PANSS total score) were randomized to either continue with risperidone treatment $2-6 \mathrm{mg} /$ day or switch to olanzapine $10-20 \mathrm{mg} /$ day for the next 10 weeks. During this third study period, the use of benzodiazepine/hypnotics/anxiolytics was permitted for the treatment of anxiety or insomnia as clinically indicated. In addition, patients who had been receiving a stable dose of an antidepressant or mood stabilizer for $\geq 30$ days prior to the initiation of the study could continue on these medications at a stable dose. Further details of the parent trial are available elsewhere. ${ }^{8}$

Figure 1 gives a graphical depiction of the study design. The three study cohorts included the early responders $(n=144)$ and the two groups of early nonresponders $(n=378)$ who were randomized to either continue risperidone (ENR-RIS, $n=192$ ) 


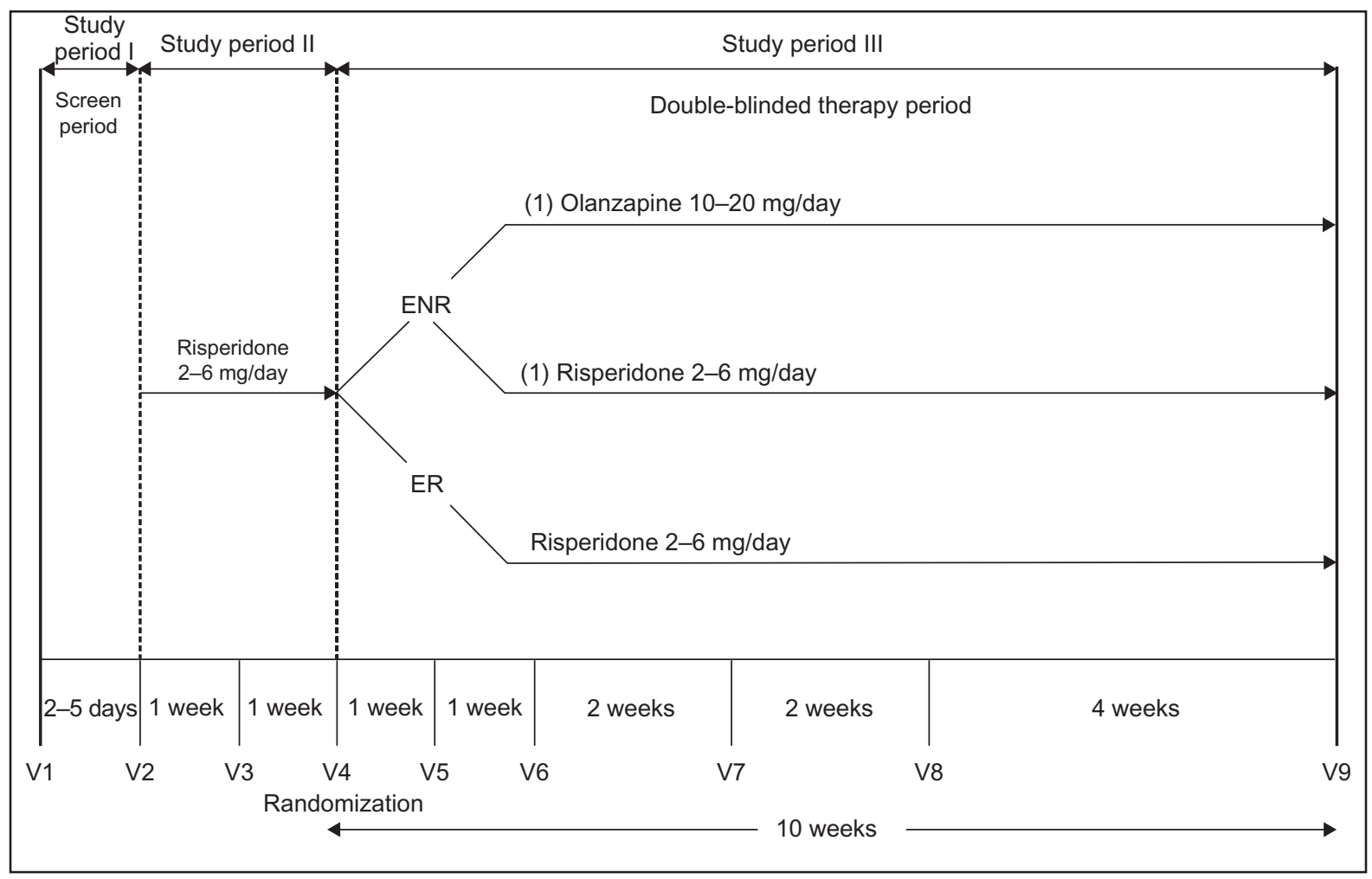

Risperidone early responder (ER)

Risperidone early non-responder (ENR)

Figure I HGMN study design.

Notes: In the parent study, 522 individuals completed two weeks of risperidone treatment (study period 2), of whom I44 were classified as early responders and 378 were classified as early nonresponders. At visit 4 , the 378 early nonresponders were randomized in a I:I fashion to continue treatment with risperidone (ENR-RIS; $n=192$ ) or switch treatment to olanzapine (ENR-OLZ; $\mathrm{n}=186$ ).

Abbreviations: $\mathrm{mg}$, milligrams; V, visit; ENR-RIS, early nonresponders to risperidone randomized to continue treatment with risperidone; ENR-OLZ, early nonresponders to risperidone randomized to switch to olanzapine.

or switch to olanzapine (ENR-OLZ, $\mathrm{n}=186$ ). Comparisons between early responders and early nonresponders contrasted only the individuals who were treated with risperidone over the 12 weeks of study periods 2 and 3, and the patients randomized to olanzapine were excluded from this comparison. Comparison between the ENR-RIS and ENR-OLZ cohorts were evaluated only over the 10 weeks of study period 3 .

Approximately 17\% (106/628) of enrolled patients discontinued risperidone treatment before the end of study period 2. Of the 522 patients who completed the initial two weeks of risperidone treatment (study period 2), 72.4\% were classified as early nonresponders, and $27.6 \%$ were classified as early responders. A similar portion of patients in each of the three cohorts discontinued study period $3: 30.6 \%$ of the early responder group, $30.2 \%$ of the ENR-RIS group, and $32.3 \%$ of the ENR-OLZ group. ${ }^{8}$

\section{Cost-effectiveness analysis}

The US Public Health Service Task Force on Cost-Effectiveness in Health and Medicine has recommended evaluating different health states based on quality-adjusted life year (QALY) ratings, where a year of life is rated on a scale from 0 (worst possible health) to 1 (perfect health), as evaluated by members of the general public. ${ }^{14}$ The ratings at specific points in time are known as utilities. QALYs are calculated from utilities by weighting the utilities according to time spent (years) at each health state and summing across the full time period. In this analysis, the timeframe was limited to the 12-week study period.

The methods used for this cost-utility analysis mirrored those from a previously published study. ${ }^{15}$ In the current analysis, we used disease-specific utility weights for eight health states (based on PANSS scores) identified in previously published research. ${ }^{16,17}$ Lenert et al identified positive, negative, and cognitive symptoms through factor analysis, and then used cluster analysis to identify eight health states from the symptom factors. Using a standard gamble methodology, utility scores were identified for the eight health states and ranged from 0.42 to 0.88 (see Table 1). In addition, Lenert et al developed disutilities for five common adverse events, ie, weight gain (0.959), orthostatic hypotension 
Table I Health state definitions

\begin{tabular}{|c|c|c|c|c|c|c|c|}
\hline \multirow[t]{2}{*}{ Disease state } & \multicolumn{3}{|c|}{ PANSS factor ${ }^{\mathrm{a}, \mathrm{b}}$} & \multirow[t]{2}{*}{ Severity $^{b}$} & \multirow[t]{2}{*}{ Dominant symptoms } & \multirow[t]{2}{*}{ Utility } & \multirow[t]{2}{*}{ Cost/month (2008) } \\
\hline & Negative & Positive & Cognitive & & & & \\
\hline I & $<2.1$ & $<2.7$ & $<2.9$ & Mild & & 0.88 & $\$ 2359$ \\
\hline 2 & $2.1-3.4$ & $<2.7$ & $<2.9$ & Moderate & Negative & 0.75 & $\$ 3323$ \\
\hline 3 & $<3.4$ & $<3.9$ & $<2.9$ & Moderate & Positive and negative & 0.74 & $\$ 5603$ \\
\hline 4 & $>3.4$ & $<3.9$ & $<2.9$ & Severe & Negative & 0.63 & $\$ 6556$ \\
\hline 5 & $<3.4$ & $<2.7$ & $>2.9$ & Severe & Positive and cognitive & 0.65 & $\$ 7|7|$ \\
\hline 6 & $>3.4$ & $<3.4$ & $>2.9$ & Severe & Negative and cognitive & 0.53 & $\$ 7296$ \\
\hline 7 & $<3.4$ & $>3.9$ & & Severe & Positive & 0.62 & $\$ 8182$ \\
\hline 8 & $>3.4$ & $>3.9$ & & Extremely severe & & 0.42 & $\$ 9951$ \\
\hline
\end{tabular}

Note: ${ }^{a}$ Mean of pertinent items on a 0-6 scale; bfrom Lenert et al ${ }^{17}$; cupdated from 1997 to 2008 US dollars using the medical component of the consumer price index (ratio of 384.943/239.1 = 1.610).

Abbreviation: PANSS, Positive and Negative Syndrome Scale.

(0.912), akathisia (0.898), pseudoparkinsonism (0.888), and tardive dyskinesia (0.857). At each assessment, a patient's total utility was assessed using the multiplicative calculation of total utility $=($ PANSS health state utility $) \times($ weight gain disutility) $\times$ (orthostatic hypotension disutility) $\times($ akathisia disutility $) \times($ pseudoparkinsonism disutility $) \times($ tardive dyskinesia disutility). ${ }^{16}$ Instances of adverse events were identified from spontaneously reported adverse events or prespecified definitions from the clinical trial protocol. ${ }^{8}$ Weight gain was identified by $a \geq 7 \%$ increase in baseline body weight, orthostatic hypotension by changes in supine to standing blood pressure and pulse, pseudoparkinsonism by the SimpsonAngus Scale ${ }^{18}$ or anticholinergic medication use, akathisia by the Barnes Akathisia Scale, ${ }^{19}$ and tardive dyskinesia by the Abnormal Involuntary Movement Scale. ${ }^{20}$ Once a patient reported an adverse event in the post-treatment period, it was assumed to continue for the remainder of the study period.

Building on these PANSS-based health states further, Mohr et $\mathrm{al}^{21}$ estimated monthly treatment costs for the eight different health states in 1997 US dollars using data from a naturalistic cost and outcomes trial. ${ }^{22}$ We updated these costs to 2008 US dollars using the medical portion of the consumer price index (see Table 1). The medication acquisition cost of olanzapine and risperidone were estimated using the least expensive combination of average wholesale price doses. ${ }^{23}$ To reflect the discounts and rebates affecting patients whose medication costs would have been paid by Medicaid, average wholesale price was further discounted by $25 \% .{ }^{24}$ Total cost was estimated as the sum of inflation-adjusted monthly cost for the different health states (nonmedication cost) and study drug acquisition costs (medication cost).

The incremental cost-effectiveness ratio identifies the cost in dollars for each unit of increase effectiveness gained. The cost per QALY gained is an important standard in cost-effectiveness research. The incremental cost-effectiveness ratio was to be calculated if one treatment was both more expensive and more effective. Alternatively, if one treatment was both more effective and less costly, it would be considered the dominant treatment, and an incremental cost-effectiveness ratio would not be calculated.

\section{Statistical methods}

Analysis of baseline characteristics, utilities, costs, and cost-effectiveness were performed for both of the group comparisons of interest, ie, risperidone early responders versus risperidone early nonresponders (excluding patients randomized to olanzapine) and early nonresponders randomized to continue with risperidone (ENR-RIS) versus switch to olanzapine (ENR-OLZ). Baseline characteristics were compared using $t$-tests for continuous variables and Chi-square tests for categorical variables. The mixed model for repeated measures was used to compare the total utility difference between treatment groups over time. The mixed model for repeated measures included a priori selected covariates for gender, race, diagnosis type, age at illness onset, investigator, as well as baseline PANSS positive, negative, and cognitive scores, and baseline utility category. Reported values represent the predicted mean values (ie, SAS least squares means) after adjusting for these covariates.

Monthly treatment costs were summed across the study period, with missing values imputed using the last observation carried forward method. Only patients with complete baseline covariate information and at least one post-baseline outcome measure were included in the analytical sample. A propensity score was calculated using logistic regression with the a priori selected covariates from the mixed model for repeated measures. An analysis of covariance adjusting for the propensity score was used to compare the total cost, 
nonmedication cost, and medication cost of the two treatment groups. Due to the skew in the cost data, a sensitivity analysis was conducted assessing mean cost differences using a propensity-score stratified, bootstrap resampling approach. ${ }^{25}$ The bootstrap resampling provides for a test of mean cost differences without making strong distributional assumptions, while the propensity stratification incorporates adjustment for the a priori set of baseline covariates.

Cost effectiveness was assessed based on the incremental cost-effectiveness ratio, defined as the group difference in mean costs divided by the group difference in utility. The variability of the incremental cost-effectiveness ratio estimate was generated by bootstrap resampling (5000 iterations) of the cost and utility values simultaneously and examining the bootstrap distribution over the quadrants of the cost-effectiveness plane. The degree of dominance criteria ${ }^{26}$ was then used to judge the strength of the observed incremental cost-effectiveness ratio. According to these criteria, if $95 \%$ of the bootstrap distribution of the incremental cost-effectiveness ratio is in the dominant quadrant (more effective and less costly), then "strict" dominance has been obtained. Alternatively, if only $50 \%$ of the bootstrap distribution of the incremental cost-effectiveness ratio is in the dominant quadrant (more effective and less costly), but $90 \%$ is in the three more effective or less costly quadrants, then the lesser criteria of "some" dominance has been obtained. All data analyses were completed using SAS version 9.1.3.

\section{Results}

The results are reported in two subsections reflecting parallel comparisons underlying the stated objectives: risperidone early responders versus risperidone early nonresponders and early nonresponders randomized to continue with risperidone (ENR-RIS) versus switch to olanzapine (ENR-OLZ). In the tables and figures, the results are juxtaposed to aid in contrasting the results from the two analyses.

\section{Early responders versus early nonresponders to risperidone}

This first analysis contrasts those who had an early response to risperidone $(n=139)$ versus those who did not have an early response to risperidone and were randomized to continue treatment with risperidone $(\mathrm{n}=188)$. In Table 2 , it can be seen that the population had an average age in their early 40s, average onset of illness in their mid-20s, most were male, the majority were either Caucasian or AfricanAmerican, and, on average, they had more severe positive than negative or cognitive symptoms of schizophrenia. At the beginning of treatment with risperidone, the early responders had significantly higher levels of positive symptoms and lower total utility scores than early nonresponders.

Total utility scores separated significantly between the early nonresponder and early responder groups at week 1 and remained significantly different across the treatment period (see Figure 2A). On average, the total utility score was 0.835 for the early responder group and 0.780 for the early nonresponder group $(P<0.001)$. Although only for a period of 12 weeks, this difference represents a gain of 4.6 quality-adjusted life days or 0.013 QALYs.

Over the 12-week study period (study periods 2 and 3), the estimated mean total cost of treating early nonresponders was significantly higher than for treating early responders

Table 2 Baseline characteristics of patients

\begin{tabular}{|c|c|c|c|c|}
\hline Variable & $\begin{array}{l}\text { ER-RIS } \\
(n=139)\end{array}$ & $\begin{array}{l}\text { ENR-RIS } \\
(n=188)\end{array}$ & $\begin{array}{l}\text { ENR-OLZ }^{a} \\
(n=179)\end{array}$ & $\begin{array}{l}\text { ENR-RIS }^{a} \\
(n=188)\end{array}$ \\
\hline Age, mean (SD) & 42.I (10.9) & $41.9(11.3)$ & $42.0(11.0)$ & $42.0(11.3)$ \\
\hline Age at onset, mean (SD) & $26.3(10.7)$ & $25.7(10.2)$ & $24.5(8.8)$ & $25.7(10.2)$ \\
\hline \multicolumn{5}{|l|}{ Race } \\
\hline African-American, \% (n) & $37.4 \%(52)$ & $47.3 \%(89)$ & $46.4 \%(83)$ & $47.3 \%(89)$ \\
\hline Caucasian, \% (n) & $51.8 \%(72)$ & $42.6 \%(80)$ & $43.6 \%(78)$ & $42.6 \%(80)$ \\
\hline Other, \% (n) & $10.8 \%(15)$ & $10.1 \%(19)$ & $10.0 \%(18)$ & $10.1 \%(19)$ \\
\hline Gender: Male, \% (n) & $59.7 \%(83)$ & $59.6 \%(112)$ & $64.8 \%(116)$ & $59.6 \%(112)$ \\
\hline PANSS total, mean (SD) & $63.8(14.3)$ & 61.1 (12.9) & $55.0(13.8)$ & $54.0(14.9)$ \\
\hline Utility total score, mean (SD) & $0.74(0.07)^{*}$ & $0.76(0.10)^{*}$ & $0.78(0.09)$ & $0.77(0.10)$ \\
\hline \multicolumn{5}{|l|}{ PANSS subscales ${ }^{16}$} \\
\hline Positive, mean (SD) & $3.1(0.64)^{*}$ & $2.7(0.64)^{*}$ & $2.4(0.74)$ & $2.3(0.79)$ \\
\hline Negative, mean (SD) & $2.2(0.74)$ & $2.1(0.86)$ & $1.9(0.74)$ & $2.0(0.83)$ \\
\hline Cognitive, mean (SD) & $1.9(0.58)$ & $1.9(0.74)$ & I.8 (0.63) & I.7 (0.70) \\
\hline
\end{tabular}

Note: $* P<0.05$; ${ }^{B}$ Baseline for the ENR-OLZ and ENR-RIS comparison was at the time or randomization (week 2 of treatment).

Abbreviations: ER-RIS, early responder to risperidone; ENR-RIS, early nonresponder to risperidone randomized to continue treatment with risperidone; ENR-OLZ, early nonresponder to risperidone randomized to switch to olanzapine; SD, standard deviation; PANSS, Positive and Negative Syndrome Scale. 


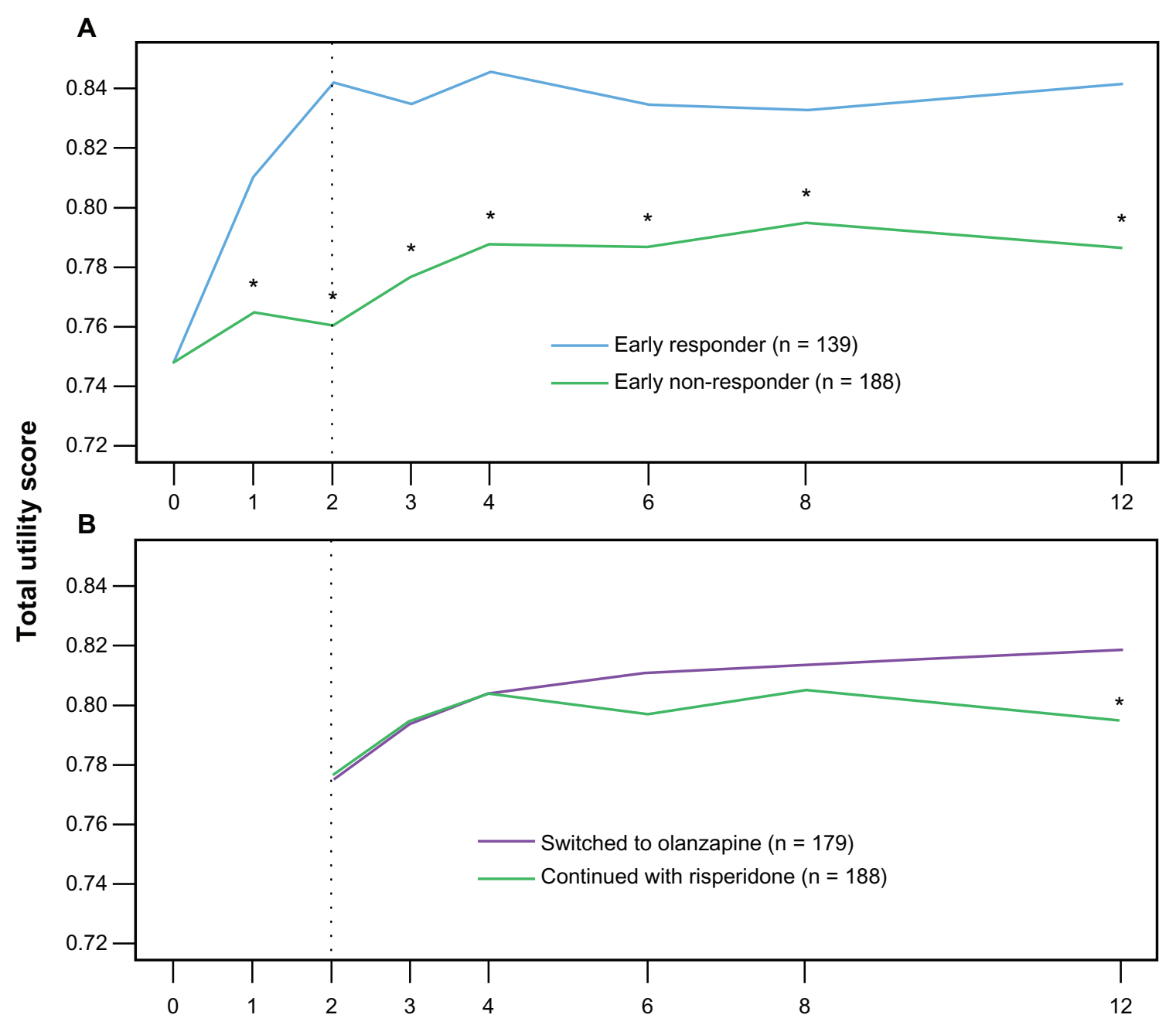

Figure 2 Total utility change over time. A) early responders versus early nonresponders over study periods 2 and 3 . B) ENR-RIS versus ENR-OLZ over study period 3. Utility scores vary from 0 (death) to I (perfect health). The $y$-axis is restricted in range from 0.72 to 0.85 to highlight the pattern of change over time.

Note: $* P<0.05$.

Abbreviations: ENR-RIS, early nonresponders to risperidone randomized to continue treatment with risperidone; ENR-OLZ, early nonresponders to risperidone randomized to switch to olanzapine.

$(\$ 9951 \pm \$ 3626$ versus $\$ 7974 \pm \$ 1886$; difference $=\$ 1977$, $P<0.001)$. The differences were driven primarily by nonmedication costs $(\$ 9528 \pm \$ 3610$ versus $\$ 7560 \pm \$ 1864$; difference $=\$ 1969, P<0.001)$, with study medication costs not differing significantly between the early nonresponder and early responder groups ( $\$ 422 \pm \$ 86$ versus $\$ 414 \pm \$ 100$; difference $=\$ 8, P=0.278$ ).

The early responders had significantly greater improvements in total utilities and significantly lower total treatment costs, resulting in early response being considered dominant. Figure 3A gives the bootstrap estimates of both the total utility changes and total cost changes. With nearly every estimate falling within the southeast quadrant, this clearly fits the definition of strict dominance (at least 95\% of estimates falling in the more effective and less costly quadrant). ${ }^{26}$

\section{Early nonresponders randomized to ENR-RIS or ENR-OLZ}

This second analysis was a randomized comparison. In Table 2 it can be seen that, at the time of randomization, the ENRRIS and ENR-OLZ groups had similar characteristics. At endpoint, total utility scores were higher for the ENR-OLZ group than for the ENR-RIS group ( $P=0.029$, see Figure 2B). The average total utility score for study period 3 was 0.808 for the ENR-OLZ group and 0.799 for the ENR-RIS group $(P=0.197)$. This difference represents a gain of 0.6 quality adjusted life days over the 10 -week period of study 3 .

The total cost was not significantly different for the ENRRIS and ENR-OLZ treatment groups $(\$ 7989 \pm \$ 3058$ versus $\$ 7875 \pm \$ 3244$; difference $=\$ 114, P>0.05$ ) over study period 3. However, the nonmedication costs $(\$ 7621 \pm \$ 3044$ versus $\$ 6634 \pm \$ 3233$; difference $=\$ 987, P<0.001$ ) 


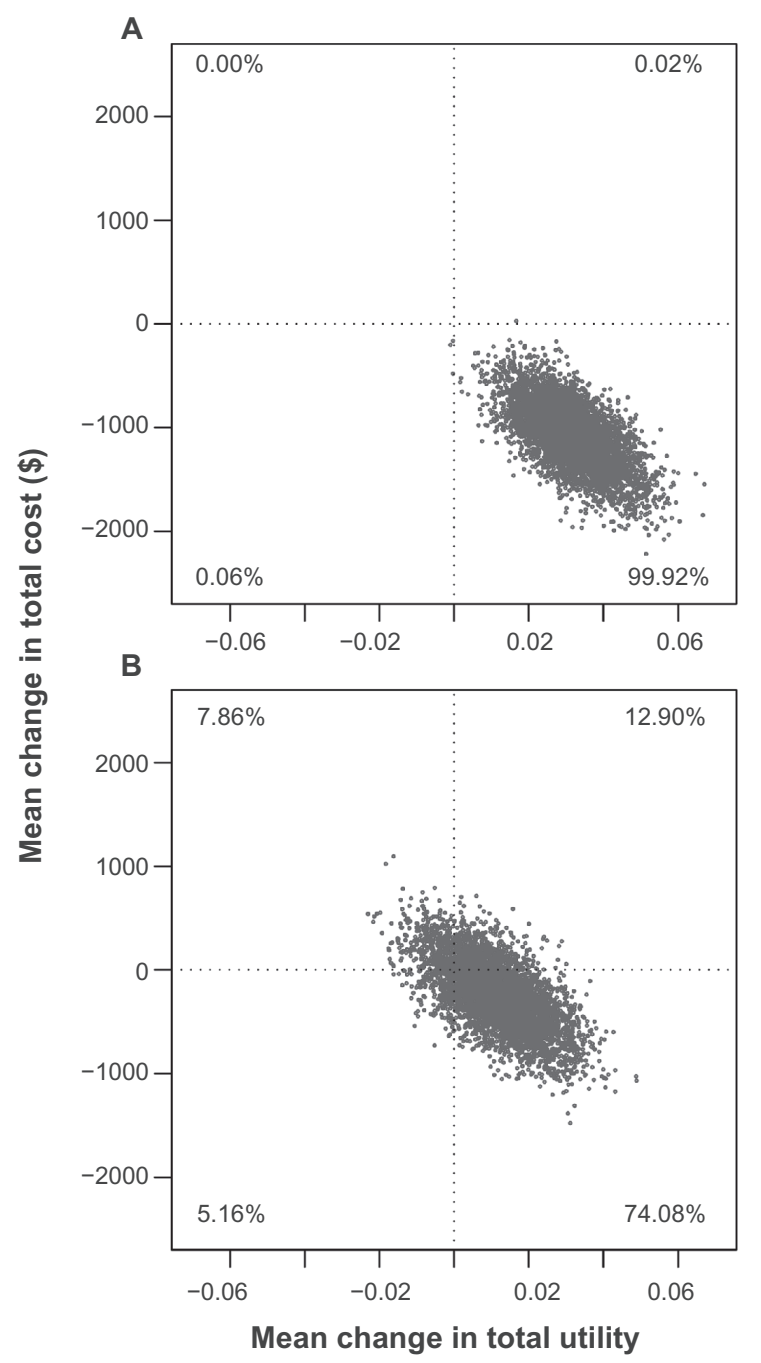

Figure 3 Distribution of 5000 bootstrap estimates of the incremental cost and effectiveness. A) Early responders versus early nonresponders comparison met criteria for strict dominance ( $95 \%$ of the bootstrap distribution in the more effective and less costly quadrant) and B) ENR-OLZ versus ENR-RIS comparison met criteria for some dominance ( $50 \%$ of the bootstrap distribution in the dominant quadrant with $90 \%$ in the three more effective or less costly quadrants).

Abbreviations: ENR-RIS, early nonresponders to risperidone randomized to continue treatment with risperidone; ENR-OLZ, early nonresponders to risperidone randomized to switch to olanzapine.

were significantly lower for the ENR-OLZ group, but were largely offset by a significantly higher study medication cost $(\$ 368 \pm \$ 81$ versus $\$ 1241 \pm \$ 253$; difference $=\$ 873$, $P \leq 0.001)$ for patients treated with olanzapine relative to those treated with generic risperidone.

Although the point estimate for the incremental cost-effectiveness ratio between the ENR-RIS and ENR-OLZ groups fell in the dominant quadrant (greater effectiveness and lower cost), the variability of this estimate did not meet the definition of strict dominance. In Figure 3B, it can be seen that the criteria of Obenchain et al for some dominance is met, given that $>50 \%$ of the estimates fall in the dominant quadrant and $>90 \%$ fall in the three more effective or less costly quadrants. ${ }^{26}$

\section{Discussion}

In this cost-effectiveness analysis, the treatment of early responders was found to be more cost-effective than the treatment of early nonresponders. The treatment of patients who were early responders to risperidone was associated with a cost saving of nearly $\$ 2000$ per patient over the 12 -week period as compared with the treatment of patients who were early nonresponders to risperidone. In addition, differences in total utility scores between early responders and early nonresponders to risperidone were significantly different by week 1 , and the difference continued over the full 12-week treatment period. Using only the 12-week study period as the timeframe resulted in 0.013 QALYs gained (out of a possible 0.23 for 12 weeks). In cost-effectiveness vernacular, the early responder is considered a dominant choice over the early nonresponder but, beyond clinical acumen for matching patients with treatments, a clinician cannot choose which individuals will be early responders to a specific antipsychotic medication.

The findings also suggest that switching the antipsychotic for early nonresponders from risperidone to olanzapine after only two weeks of treatment may represent a cost-effective treatment strategy. Early nonresponders to risperidone who were switched to olanzapine had better total utility scores after 10 weeks of treatment (at endpoint). A significant reduction in nonmedication health care costs was offset by the higher medication acquisition cost of olanzapine relative to generic risperidone. The QALYs gained during the 10-week period were quite small, representing only 0.6 of a quality-adjusted life day per patient. In cost-effectiveness research, interpreting the dominance of a treatment when the point estimate is both more effective and less costly, but when only one (in this case the effectiveness) is statistically significant poses a challenge. Obenchain et al recommended considering the statistical significance of both the cost and effectiveness simultaneously and proposed three different levels of dominance. ${ }^{26}$ Our result, as displayed in Figure 3B, meets their criteria for the lowest level of dominance, ie, some dominance. Although not nearly as compelling as the results for the early responders relative to the early nonresponders to risperidone, switching patients who are early nonresponders to risperidone to treatment with olanzapine may represent a cost-effective strategy.

Our finding that early responders to atypical antipsychotics had better outcomes in terms of QALYs is consistent with the findings of other schizophrenia studies contrasting early responders and early nonresponders to various antipsychotic medications. 
Previous studies have shown the implications of poor early response, not only in terms of poor response of core symptoms of schizophrenia, ${ }^{3,4,6,7}$ but also in terms of reduced clinician-assessed quality of life, clinician-assessed functioning, patient-reported well-being, clinician-assessed depression, ${ }^{9}$ patient-reported functioning, and patients' perceptions of medication benefits. ${ }^{5}$ Previous studies have also found that health care costs were just over $\$ 2000$ higher during an eight-week period for early nonresponders relative to early responders, ${ }^{5}$ a finding that is very similar to the cost finding in the current analysis. This growing body of research questions the prior recommendation from consensus treatment guidelines to wait 4-8 weeks before changing antipsychotic regimens for individuals who do not show a minimal early response to the initial antipsychotic medication.

Given the broad array of differences in outcomes between early responders and early nonresponders, one of the sobering findings in this study was the large percentage of early nonresponders $(72 \%, 378 / 522)$. Although some of these early nonresponders do later respond to treatment, the majority (approximately 80\%) do not adequately respond. ${ }^{4,8}$ In the HGMN primary analysis, ${ }^{8}$ as well as in this cost-effectiveness analysis, switching medication to olanzapine showed a small but statistically significant improvement in outcomes at endpoint relative to continuing on risperidone. However, the patients who were switched to the alternative therapy did not have outcomes that were nearly as favorable as those for the early responders. These early nonresponders may represent a more difficult-totreat population, but more research investigating these initial poor responders is needed.

\section{Limitations}

The current analysis was restricted to patients treated with risperidone (early responders and early nonresponders), with early nonresponders randomized in a double-blind manner to continue on risperidone or switch to olanzapine. Therefore, our results may not generalize to other combinations of antipsychotic medication switches.

The total utility scores used in this analysis were calculated from symptom scores and adjusted for adverse event disutilities. The total utility score represents a weighted measure of both the efficacy and adverse events of the different medications as rated by a representative sample of individuals in the US. ${ }^{16}$ QALYs have been recommended for use in cost-effectiveness research with antipsychotics because they allow direct comparison of therapies across a number of domains and can be understood in terms of willingness to buy a unit for this outcome. ${ }^{27}$ An additional strength of the PANSS-based QALY ratings ${ }^{16,17}$ is that they were based on health domains that are specifically affected by psychotic illnesses and, therefore, may be more sensitive to important changes in symptoms of schizophrenia than generic measures of quality of life. ${ }^{27}$

Our simplifying assumption that once an adverse event occurred it did not resolve during the study may have served to underestimate the total utility slightly. However, given the short duration of the trial and the size of adverse event disutilities relative to the disease state utilities, we anticipate this assumption had a negligible effect on the final results.

A challenge in conducting cost-effectiveness research using multinational clinical trials is that the patterns of resource use and the cost of those resources vary greatly by geography. In some countries, psychiatric hospitalization is inexpensive relative to the cost of medications, whereas in the US, psychiatric hospitalization represents one of the most expensive aspects of care. However, the mean duration of psychiatric hospitalization in the US is relatively short compared with some countries (eg, Brazil and Japan). To circumvent this issue in our analysis, cost was assigned based on previously identified monthly costs in the US for the different health states, ${ }^{21}$ rather than direct collection of and costing of resource use, which was not included in the HGMN trial. Costing based on health states has been used previously ${ }^{15}$ and involves some additional assumptions, including that health states are the main driver of costs and that the previously identified costs for the health states generalize to this patient population.

Finally, although the statistical analysis was adjusted for several important baseline variables, the results of the comparison between early responders and early nonresponders to risperidone are still open to biases from other confounders not accounted for in the statistical model. The patients who were early responders to risperidone may have differed in other significant ways from those who were early nonresponders.

\section{Conclusion}

Treatment of early responders was significantly more costeffective than the treatment of early nonresponders to atypical antipsychotic therapy (risperidone). Early nonresponders to antipsychotic therapy, following only two weeks of treatment, appear to represent a group of patients who are more challenging to treat and tend to incur greater health care costs. In this study, switching early nonresponders from risperidone to olanzapine at two weeks provided significantly lower nonmedication health care costs, significantly higher medication costs, and modest improvements in utility scores. One potential approach to the cost-effective treatment of 
early nonresponders may be to switch their medications after two weeks. Further research into the effectiveness of switching paradigms and alternative treatments is needed.

\section{Acknowledgments}

The authors would like to thank Michael D Stensland, PhD of Agile Outcomes Research, In. and Susan L Dennett, PhD of Strategic Health Outcomes, Inc for medical writing support on behalf of Eli Lilly and Company.

\section{Disclosure}

This study was funded by Eli Lilly and Company. HA, $\mathrm{XP}, \mathrm{DF}, \mathrm{VS}, \mathrm{SKW}$, and BK are all full-time employees and minor stockholders of Lilly. JK serves as a consultant and is on the speaker's bureau for Astra-Zeneca (speaker), Bristol-Myers Squibb (speaker), Cephalon, Dainippon Sumitomo, GlaxoSmithKline, Intracellullar Therapeutics, Janssen (speaker), Johnson \& Johnson, Eli Lilly and Company (speaker), Otsuka America Pharmaceutical Inc (speaker), Pfizer, PGxHealth, Proteus, Takeda, Vanda, and Wyeth.

\section{References}

1. Lehman AF, Lieberman JA, Dixon LB. Practice guideline for the treatment of patients with schizophrenia, second edition. Am J Psychiatry. 2004;161(9): 1-56.

2. Canadian Psychiatric Association. Clinical practice guidelines. Treatment of schizophrenia. Can J Psychiatry. 2005;50(13 Suppl 1):S7-S57.

3. Correll CU, Malhotra AK, Kaushik S, McMeniman M, Kane JM. Early prediction of antipsychotic response in schizophrenia. Am J Psychiatry. 2003;160(11):2063-2065.

4. Kinon BJ, Chen L, Ascher-Svanum H, et al. Predicting response to atypical antipsychotics based on early response in the treatment of schizophrenia. Schizophr Res. 2008;102(1-3):230-240.

5. Ascher-Svanum H, Nyhuis AW, Faries DE, et al. Clinical, functional, and economic ramifications of early nonresponse to antipsychotics in the naturalistic treatment of schizophrenia. Schizophr Bull. 2008;34(6): 1163-1171.

6. Agid O, Kapur S, Arenovich T, Zipursky RB. Delayed-onset hypothesis of antipsychotic action: A hypothesis tested and rejected. Arch Gen Psychiatry. 2003;60(12):1228-1235.

7. Leucht S, Busch R, Hamann J, Kissling W, Kane JM. Early-onset hypothesis of antipsychotic drug action: A hypothesis tested, confirmed and extended. Biol Psychiatry. 2005;57(12):1543-1549.

8. Kinon BJ, Chen L, Ascher-Svanum H, et al. Early response to antipsychotic drug therapy as a clinical marker of subsequent response in the treatment of schizophrenia. Neuropsychopharmacology. 2010;35(2): 581-590.

9. Kinon BJ, Chen L, Ascher-Svanum H, et al. Challenging the assumption that improvement in functional outcomes is delayed relative to improvement in symptoms in the treatment of schizophrenia. Schizophr Res. 2010;118(1-3):176-182.

ClinicoEconomics and Outcomes Research

\section{Publish your work in this journal}

ClinicoEconomics \& Outcomes Research is an international, peerreviewed open-access journal focusing on Health Technology Assessment, Pharmacoeconomics and Outcomes Research in the areas of diagnosis, medical devices, and clinical, surgical and pharmacological intervention. The economic impact of health policy and health systems
10. American Psychiatric Association. Diagnostic and Statistical Manual of Mental Disorders DSM-IV-TR Fourth Edition. New York, NY: American Psychiatric Publishing Inc; 2000.

11. Overall JE, Gorham DR. The Brief Psychiatric Rating Scale. Psychol Rep. 1962;10:799-812.

12. Kay SR, Opler LA, Fiszbein A. Positive and Negative Syndrome Scale (PANSS) User's Manual. North Tonawanda, NY: Multi-Health Systems Inc; 2000

13. Guy W. ECDEU Assessment Manual for Psychopharmacology. In: Publication ADM 76-338. Revised. Rockville, MD: US Department of Health, Education, and Welfare; 1976.

14. Gold MR, Siegel JE, Russell LB, Weinstein MC. Cost-Effectiveness in Health and Medicine. 1st ed. New York, NY: Oxford University Press; 1996.

15. Kane JM, Kim E, Kan HJ, et al. Comparative utility of aripiprazole and haloperidol in schizophrenia: Post hoc analysis of two 52-week, randomized, controlled trials. Appl Health Econ Health Policy. 2009; 7(2):109-119.

16. Lenert LA, Sturley AP, Rapaport MH, et al. Public preferences for health states with schizophrenia and a mapping function to estimate utilities from positive and negative symptom scale scores. Schizophr Res. 2004;71(1):155-165.

17. Lenert LA, Sturley AP, Rapaport MH, et al. Corrigendum to "Public preferences for health states with schizophrenia and a mapping function to estimate utilities from positive and negative symptom scale scores". Schizophr Res. 2005;80(1):135-136.

18. Simpson GM, Angus JW. A rating scale for extrapyramidal side effects. Acta Psychiatr Scand Suppl. 1970;212:11-19.

19. Barnes TR. A rating scale for drug-induced akathisia. Br J Psychiatry. 1989; 154:672-676.

20. National Institute of Mental Health. Abnormal Involuntary Movement Scale (AIMS). In: ECDEU Assessment Manual for Psychopharmacology, Revised. Rockville, MD: US National Institute of Health; 1976.

21. Mohr PE, Cheng CM, Claxton K, et al. The heterogeneity of schizophrenia in disease states. Schizophr Res. 2004;71(1):83-95.

22. Mahmoud RA, Engelhart LM, Janagap CC, Oster G, Ollendorf D. Risperidone versus conventional antipsychotics for schizophrenia and schizoaffective disorder: Symptoms, quality of life and resource use under customary clinical care. Clin Drug Investig. 2004;24(5): $275-286$.

23. Analysource Data [Website on the Internet]. AWP Cost of Antipsychotics. Available from: http://www.analysource.com. Accessed March 31, 2008.

24. Rosenheck RA, Leslie DL, Sindelar J, et al. Cost-effectiveness of second-generation antipsychotics and perphenazine in a randomized trial of treatment for chronic schizophrenia. Am J Psychiatry. 2006; 163(12):2080-2089.

25. Faries D, Leon A, Haro J, Obenchain R. Analysis of Observational Health Care Data Using SAS. Cary, NC: SAS Press; 2010.

26. Obenchain RL, Robinson RL, Swindle RW. Cost-effectiveness inferences from bootstrap quadrant confidence levels: Three degrees of dominance. J Biopharm Stat. 2005;15(3):419-436.

27. Polsky D, Doshi JA, Bauer MS, Glick HA. Clinical trial-based cost-effectiveness analyses of antipsychotic use. Am J Psychiatry. 2006; 163(12):2047-2056

organization also constitute important areas of coverage. The manuscript management system is completely online and includes a very quick and fair peer-review system, which is all easy to use. Visit http://www.dovepress.com/testimonials.php to read real quotes from published authors. 